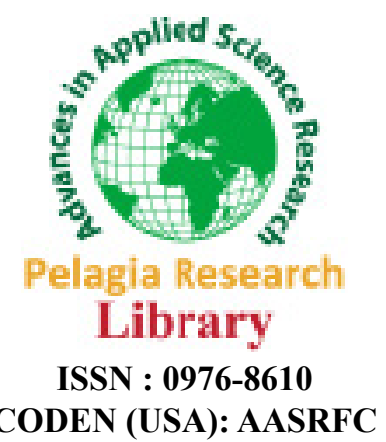

\title{
Analyzing the Conceptual Model for Exploratory Testing Framework using PLS-SEM
}

\author{
Thangiah Murugan, Shuib Basri, and Dhanapal Durai Domnic \\ Universiti Teknologi Petronas, Bandar Sri Iskandar 32610, Seri Iskandar, Perak, Malaysia
}

\begin{abstract}
Small and Medium size Software Enterprises (SME's) often faces many challenges in the pursuit of developing quality software due to various factors such as limited resources, time and complexity, cost, frequent changes in the requirement by the stakeholder due to the agile development process where customer satisfaction is paramount. Even though the testing activities carried out in a traditional way but still there are many projects exposed to vulnerability and other critical issues which goes unnoticed. Exploratory Testing is an approach which helps to solve this issue by identifying the bugs which can be fixed before being delivered to the stakeholder. So far there is no standard approach available for Exploratory Testing. Based on the previous research work and analysing various literatures, a new conceptual framework has been developed. The proposed conceptual framework for Exploratory Testing, is to be analysed using Partial Least Squares Structural Equation Modelling (PLS-SEM). The reliability and validity of the research framework is being assessed using the measurement model in PLS-SEM.
\end{abstract}

Keywords: Conceptual framework, Exploratory testing, PLS-SEM, SMSE's.

\section{INTRODUCTION}

Small and Medium size Software Enterprises (SMEs) are becoming a cornerstone and provide a significant contribution in the worldwide industry economy for the past two decades. Software applications have now-a-days become an increasing part of life, most people have had experiences with the software applications that it did not work as expected, which resulted in loss of money, loss of time or business reputation. The development team faces increasing pressure in productivity regarding many software development principles, however, most of them agree on one common thing above all Quality [1]. Software testing is a process of finding the defects or bugs in a product to ensure that the intended product works well within its scope and boundary and meet the stakeholder's requirement [2-12]. In other words, software testing is a process of evaluating the system that provides information to the customer or stakeholder and the product functions as expected in order to ensure the quality of the product. Hence, it is essential to have good quality software.

Exploratory Testing is an approach that helps to identify unknown bugs which are missed during traditional testing activities [13-18]. In agile development process, exploratory testing plays an important role which can also be combined with other analytical risk-based testing, model-based testing and regression-averse testing [19]. Unlike scripted testing, exploratory testing is not carried out precisely according to plan and defined in advance. Exploratory testing is mostly used and recommended, though not used currently, if the requirements are incomplete and time to release the software is less [20,21].

A conceptual framework for Exploratory Testing is developed based on the literature review and quantitative study conducted by using a survey. This survey questionnaire was distributed to various small and medium size enterprises in Malaysia and the data analysis was done using Partial Least Squares Structural Equation Modeling (PLS-SEM) [4] According to Hair et al., [3] PLS-SEM has provided many advantages for the researchers who require additional 
discussion to clarify the reason behind the choice [3,7]. The most prominent justification for using PLS-SEM are attributed when having a small samples sizes, non-normal data, limited theory available about the applications, predictive accuracy is paramount and formatively measured constructs [3,7]. Using PLS-SEM, the analysis is done to verify the reliability and validity of the data that has not been exposed to the method. Further analysis will be carried out and reported in future works.

Using PLS-SEM, the researchers need to apply a multi-stage process which involves the determination of data collection and examination, creating outer models (indicators to latent variable) and inner models (connecting between latent variables), the real model estimation and assessment of results. This research paper focuses only on model specification which is developed based on the conceptual framework and the outer model (measurement model) evaluation is conducted. The inner model evaluation, which is not within the scope of this research paper, will be analyzed and assessed as a future work in continuation of this research.

\section{Partial Least Squares Structural Equation Modeling}

According to Hair et al., understanding the relationships between data, researchers are initially relied on univariate or bivariate analysis. However, to comprehend more complex relationships associated with current research direction, a more sophisticated and comprehensive multivariate data analysis is increasingly necessary [3]. Structural equation modeling (SEM), as a second-generation technique, is a family of statistical models that seek to explain the simultaneous modeling and its relation- ships among multiple variables [5]. SEM is also like a series of multiple regression equations, that examines the structure of inter relationships between the con- structs that are expressed in a series of equations. These equations show the entire relationships between the independent and dependent variables (constructs) involved in the analysis. The constructs are no longer differentiates between dependent and independent variables rather it distinguishes between exogenous and endogenous variables and these variables are latent factors represented by multiple variables. The endogenous variables are explained in the relationship contained in the model and the exogenous variables which are not explained by the postulated models always act as independent variables $[3,5,7]$.

\section{Model Specification}

Path models are diagrams which are used to visually display the variable relationships and the hypothesis that are examined in SEM $[3,5,7]$ and the conceptual model is shown in Figure 1. The model specification deals with

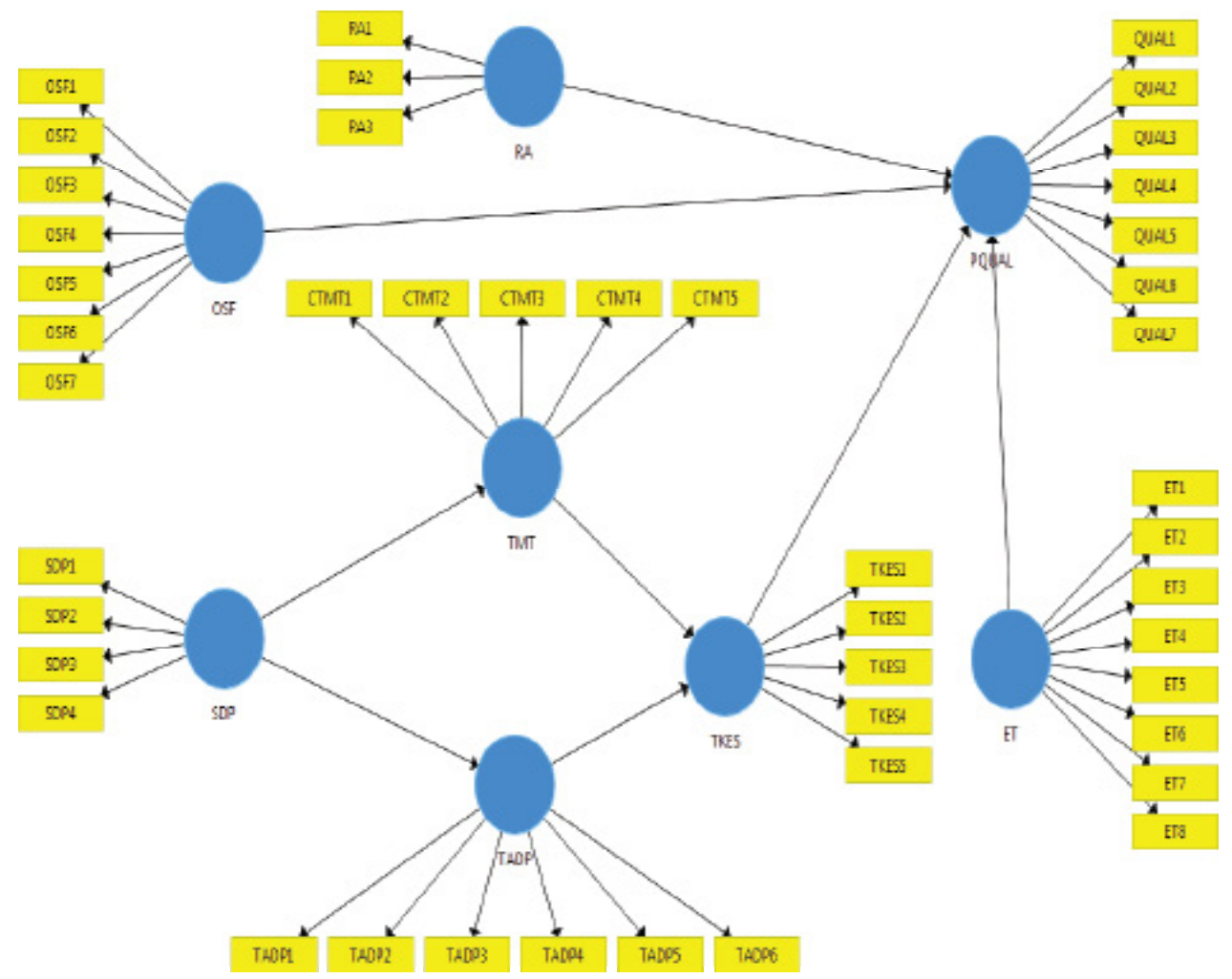

Figure 1: Conceptual model. 
construction of inner model and outer model. The relationship between the indicator variables and their corresponding constructs are evaluated by the outer model or measurement model. The inner model, also known as structural model, is used to evaluate the relationships between the con- structs. Based on this guideline, the construct is created and the indicator variables which are associated with the constructs are connected as a reflective indicator [3,5,7-9].

\section{RESEARCH MODEL}

\section{Conceptual Framework Development}

Based on the literature, a conceptual framework on Exploratory Testing is developed. It has six independent or exogenous variables, one dependent or endogenous variable and one control variable. The conceptual model for the proposed framework is created using the Smart PLS software [5]. Independent variables are key factors which should affect the product quality in the SME's. The dependent variable is the quality factor which is the essential factor for SME's to produce quality project to satisfy the stake- holders and the moderated variable will be the key factors to achieve the perceived quality.

\section{Populations and Samples}

Small and Medium size Enterprises (Software development Companies only) have been selected as the target population for this study. This population only includes SME's which are undertaking software development activities. Other SMEs which provide support services, consultancy services, selling a product, hardware, networking and installation are ignored for this study. The study was conducted at a random sample of 315 companies representing, Selangor, Kuala Lumpur and Penang in Malaysia. First a qualitative study was conducted based on interviews and observations with the SMEs. This is to ensure that those companies are involved in software development activities and to ensure that the number of employees and the annual turnover are within the range of SMEs definition specified in the SME Corporation, Malaysia [22,23].

A survey questionnaire was created based on the conceptual framework and distributed to the various small and medium size software industries. Out of 315 companies given out only 200 samples were selected, and the remaining survey was rejected for various reasons. The data collected from the questionnaire was entered in the MS- Excel and saved as .csv format which can be supported by various statistical tools to conduct reliability tests. The factors that have been identified based on the definition in the conceptual model (Figure 1) six endogenous (independent), one exogenous and the variable which influenced the software quality were operationalized and used to collect the data for this research study.

\section{Variables and Measures}

For this research study six independent variables (exogenous), were identified such as Organization Structure, Requirement analysis, testing in agile development, tester's skills knowledge and experience, testing methods and tools, and software development process. One dependent variable - Perceive Quality, and a control variable exploratory testing was used in the conceptual model. A multi-item, five-point, bipolar Likert scales that ranged from "strongly disagree" (1) to "strongly agree" (5) for all indicators were selected. The item ratings were summarized to form a summated rating scale for each independent variable. Furthermore, since this is the first study of its kind within Exploratory Testing, all the items were written specifically for this study.

\section{Measurement Model and Assessment}

According to Hair et al., the systematic assessment criteria follows two-step process with the separate assessment for measurement models and structural model [3,5]. For the evaluation of reflective measurement model, construct validity should be assessed first. The construct validity which is made up of two important components are convergent validity and discriminant validity. The convergent validity evaluates the fac- tor loadings, composite reliability and Average Variance Extracted (AVE) and the discriminant validity evaluates cross loadings, Fornell \& Larcker and HTMT.

\section{Assessment of Convergent Validity}

Convergent validity refers to as "the extent to which a measure (indicators) correlates positively with alternative measures (indicators) of the same construct" [3]. Table 1 shows the details of each constructs used in the conceptual framework and indicators associated with each construct are listed in the column name "Item". First the composite reliability is verified and any value below the threshold values of 0.7 needs to be assessed. This is established by checking the indicator reliability values. Any values in the indicator which is below 0.4 should be removed from the 
con- structs and any value between 0.4 and 0.6 can be retained if it increases the AVE and CR values. After removing the indicators from the constructs, the details of the remaining constructs and its corresponding convergent validity scores have met the threshold values for $\mathrm{CR}>0.7$ and AVE $>0.5$ respectively.

\section{Assessment of Discriminant Validity}

To ensure that individual constructs are truly distinct from each other, discriminant validity assessment should be conducted. According to Fornenell and Larcker [16], the square root of AVE in each construct must be larger than other correlation values between other constructs. Table 2 depicts the assessment of discriminant validity for this conceptual framework. The square root of the AVE of each construct which is highlighted in bold are larger than the correlation estimates of the factors. This indi- cates that all the constructs exhibit discriminant validity and are distinct from one another.

Table 3 depicts another method of assessing the discriminant validity which is provide in the PLS software also called Heterotrait-Monotrait criteria. According to Henseler et al., [18], he proposed a HTMT value between the range -1 and $1(-1<$ HTMT $<1)$ and any constructs which has the value between the range are consider that the discriminant

Table 1: Convergent validity: Construct reliability and validity.

Construct
Requirement Analysis
(RA)
Software Development
Process (SDP)
Testing Technique and
Methods (TMT)
Organizational
Structure (OS)
Testing in Agile
Development (TADP)
Tester's Knowledge,
Experience (TKES)
Exploratory Testing
(ET)
Quality (QUAL)

Criteria: Composite reliability $>0.7$, AVE $>0.5[3]$

\begin{tabular}{|c|c|c|c|c|}
\hline No of Items & Removed & CR & AVE & Alpha \\
\hline 3 & None & 0.889 & 0.727 & 0.813 \\
\hline 4 & None & 0.865 & 0.620 & 0.794 \\
\hline 5 & 1 & 0.847 & 0.587 & 0.752 \\
\hline 7 & 2 & 0.860 & 0.559 & 0.829 \\
\hline 6 & None & 0.891 & 0.580 & 0.856 \\
\hline 5 & None & 0.869 & 0.574 & 0.814 \\
\hline 7 & 2 & 0.883 & 0.573 & 0.835 \\
\hline
\end{tabular}

Note: SOS1, SOS2, TMT5, ET1 and ET2 are removed due to low loadings

Table 2: Discriminant validity [16].

\begin{tabular}{|c|c|c|c|c|c|c|c|c|}
\hline Variables & $\mathbf{1}$ & $\mathbf{2}$ & $\mathbf{3}$ & $\mathbf{4}$ & $\mathbf{5}$ & $\mathbf{6}$ & $\mathbf{7}$ & $\mathbf{8}$ \\
\hline OS & 0.742 & -- & -- & -- & -- & -- & -- & -- \\
\hline RA & 0.025 & 0.751 & -- & -- & -- & -- & -- & -- \\
\hline SDP & 0.097 & 0.109 & 0.896 & -- & -- & -- & -- & -- \\
\hline TTM & -0.011 & 0.08 & 0.347 & 0.758 & -- & -- & -- & -- \\
\hline ET & -0.011 & -0.054 & -0.061 & -0.094 & 0.748 & -- & -- & -- \\
\hline QUAL & 0.078 & -0.145 & -0.007 & -0.084 & 0.744 & 0.727 & -- & -- \\
\hline TAD & 0.068 & -0.162 & -0.081 & -0.094 & 0.286 & 0.254 & 0.73 & -- \\
\hline TKE & -0.199 & 0.032 & -0.019 & 0.046 & -0.106 & -0.183 & -0.052 & 0.74 \\
\hline
\end{tabular}

Table 3: Heterotrait Monotrait (HTMT) criterion for discriminant validity.

\begin{tabular}{|c|c|c|c|c|c|c|c|c|}
\hline Variables & $\mathbf{1}$ & $\mathbf{2}$ & $\mathbf{3}$ & $\mathbf{4}$ & $\mathbf{5}$ & $\mathbf{6}$ & $\mathbf{7}$ & $\mathbf{8}$ \\
\hline OS & -- & -- & -- & -- & -- & -- & -- & -- \\
\hline RA & 0.149 & -- & -- & -- & -- & -- & -- & -- \\
\hline SDP & 0.123 & 0.169 & -- & -- & -- & -- & -- & -- \\
\hline TTM & 0.144 & 0.13 & 0.434 & -- & -- & -- & -- & -- \\
\hline ET & 0.092 & 0.084 & 0.108 & 0.134 & -- & -- & -- & -- \\
\hline QUAL & 0.105 & 0.156 & 0.107 & 0.125 & 0.88 & -- & -- & -- \\
\hline TAD & 0.117 & 0.21 & 0.105 & 0.111 & 0.333 & 0.282 & -- & -- \\
\hline TKE & 0.248 & 0.162 & 0.08 & 0.086 & 0.158 & 0.196 & 0.085 & -- \\
\hline
\end{tabular}


validity is achieved. In this conceptual framework all the values between each construct are between the range -1 to 1 and hence the discriminant validity is achieved.

\section{DISCUSSION}

The reason to assess the measurement model is to identify the correlation between the constructs and to evaluate the relationship between the indicators. This is to guarantee that each of the constructs in the conceptual framework is distinct from the other.

Three assessments were conducted to evaluate the measurement model and they are:

i) Assessment of construct reliability,

ii) Assessment of convergent validity and

iii) Assessment of discriminant validity.

For this research study all the constructs met the minimum threshold criterion for assessment of construct reliability (Composite reliability score $>0.7$ ). Similarly, in the assessment of convergent validity, each of the constructs met the minimum requirement of AVE $>0.5$ and factor loadings of more than 0.7. For some indicator like CRA2, SOS3 the factor loadings are below the threshold values for which we have provided relevant arguments for retaining it in the final measurement model.

Fornell \& Larcker criterion and HTMT criterion is used to assess the discriminant validity of measurement model. For this research study, it was shown that each of the constructs is unique and different from each other. Having fulfilled and met the re- quirements of measurement model assessment, the next step was the assessment of structural model and these results are important for analyzing the theory developed for this conceptual framework.

\section{CONCLUSION}

In this research paper, the assessment of measurement model was conducted to evaluate the relation between the independent variable, dependent variable and the effect of moderation variables for the Exploratory Testing conceptual framework by using the Smart PLS software. The results are discussed and provided in this paper will used as a next step to conduct the structural model assessment. So far there is no research paper available to evaluate the conceptual framework for Exploratory Testing using PLS-SEM and this will be the first in the area of Software Engineering field.

\section{REFERENCES}

[1] Iacob M, Constantinescu R. Testing: First step towards software quality. Journal of Applied Quantitative Methods, 2008, 3: 3.

[2] Van der Broek R, Bonsangue MM, Chaudron M. Integrating testing into Agile software development processes, 2nd International Conference on Model-Driven Engineering and Software Development (MODELSWARD) 2014, pp: 561-574.

[3] Hair JF, Hult GTM, Ringle CM. A Premier on Partial Least Squares Structural Equation Modeling (PLS-SEM), Thousand Oaks: Sage, 2014.

[4] Ringle CM, Wende S, Becker JM. SmartPLS 3. Boenningstedt: SmartPLS GmbH, http://www.smartpls.com. 2015.

[5] Gefen D, Straub DW, Boudreau MC. Structural equation modeling and regression: Guidelines for research practice. Communications of the Association for Information Systems. 2000, 4: 1-79.

[6] Urbach N, Ahlemann F. Structural equation modelling in information systems re- search using partial least squares. Journal of Information Technology Theory and Application. 2010, 11: 5-40.

[7] Chin WW. How to Write Up and Report PLS Analyses. Handbook of Partial Least Squares. Springer Han dbooks of Computational Statistics. Springer, Berlin, Heidelberg. 2010, 655-690.

[8] Wong KK. "Handling small survey sample size and skewed dataset with partial least square path modeling. Vue: The Magazine of the Marketing Research and Intelligence Association. 2010, 20-23.

[9] Wong KK, "Review of the book Handbook of Partial Least Squares: Concepts, Methods and Applications. International Journal of Business Science and Applied Management. 2011, 6: 52-54. 
[10] Gleirscher M, Golubitskiy D, Irlbeck M. Introduction of static quality analysis in small- and medium-sized software enterprises: Experiences from technology transfer. Software Quality Journal, 2014.

[11] Johan van Zyl, Software testing in a small company: A case study. Technical Report, University of Pretoria, 2010.

[12] Shoaib L, Nadeem A, Akbar A. An empirical evaluation of the influence of human personality on exploratory software testing, Multitopic Conference, 2009. INMIC 2009. IEEE 13th International, 2009, 1: 14-15.

[13] Ayse T, Ayse B, Burak T. Implementation of a Software Quality Improvement Project in an SME: A Before and After Comparison", Proc. 35th Euromicro Conference on Software Engineering and Advanced Applications, 2009, 203-209.

[14] Jörg Henseler Christian M, Ringle Marko Sarstedt. Testing measurement invariance of composites using partial least squares, International Marketing Review, 2016, 33: 3.

[15] Hair JF, Ringle CM, Sarstedt M, PLS-SEM: Indeed, a sliver bullet. Journal of Marketing Theory and Practice, 2011, 19: 139-151.

[16] Fornell C, Larcker DF. Evaluating structural equation models with unobservable variables and measurement error. Journal of Marketing Research, 1981, 18: 39-50.

[17] Diamantopoulos, Adamantios. Judy AS, Formative vs. reflective indicators in measure development: Does the choice of indicators matter? British Journal of Management, 2006, 13: 263-282.

[18] Henseler J, Dijkstra TK, Sarstedt, Common beliefs and reality about partial least squares: Comments on Organizational Research Methods, 2014, 17: 182-209.

[19] Philip I. 3 reasons why exploratory testing is great for agile teams. 2017.

[20] James Bach. What is exploratory testing? 2017.

[21] Cem Kaner, Exploratory Testing. 2017.

[22] Chin WW. The partial least squares approach to structural equation modeling. Modern business research methods. Mahwah, NJ: Lawrence Erlbaum Associates. 1998.

[23] Economic Census 2016-Profile of SMEs, 2017. 